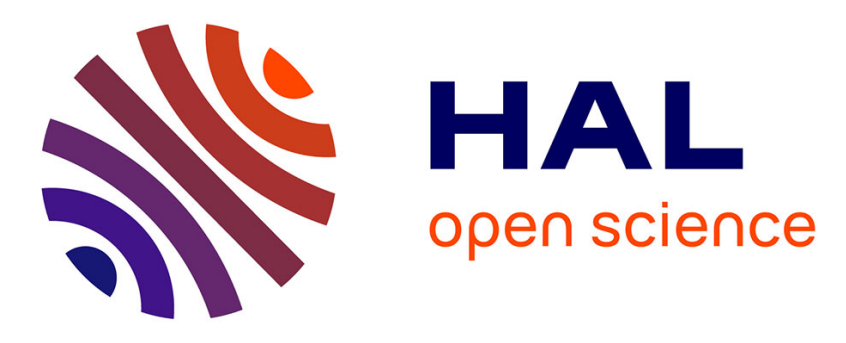

\title{
3D segmentation of coronary arteries based on advanced mathematical morphology techniques
}

Bessem Bouraoui, Christian Ronse, Joseph Baruthio, Nicolas Passat, Philippe Germain

\section{- To cite this version:}

Bessem Bouraoui, Christian Ronse, Joseph Baruthio, Nicolas Passat, Philippe Germain. 3D segmentation of coronary arteries based on advanced mathematical morphology techniques. Computerized Medical Imaging and Graphics, 2010, 34 (5), pp.377-387. 10.1016/j.compmedimag.2010.01.001 . hal01694412

\section{HAL Id: hal-01694412 \\ https://hal.univ-reims.fr/hal-01694412}

Submitted on 3 Mar 2018

HAL is a multi-disciplinary open access archive for the deposit and dissemination of scientific research documents, whether they are published or not. The documents may come from teaching and research institutions in France or abroad, or from public or private research centers.
L'archive ouverte pluridisciplinaire HAL, est destinée au dépôt et à la diffusion de documents scientifiques de niveau recherche, publiés ou non, émanant des établissements d'enseignement et de recherche français ou étrangers, des laboratoires publics ou privés. 


\title{
3D segmentation of coronary arteries based on advanced Mathematical Morphology techniques
}

\author{
B. Bouraoui ${ }^{\mathrm{a}, \mathrm{b}}$, C. Ronse ${ }^{\mathrm{a}, *}$, J. Baruthio $^{\mathrm{b}}$, N. Passat ${ }^{\mathrm{a}}$, \\ P. Germain ${ }^{\mathrm{c}}$ \\ ${ }^{\mathrm{a}}$ LSIIT UMR 7005 CNRS/UdS, Parc d'Innovation, Boulevard S. Brant, BP \\ 10413, 67412 Illkirch Cedex, France \\ ${ }^{\mathrm{b}}$ LINC UMR 7191 CNRS/UdS, 4 rue Kirschleger, 67085 Strasbourg Cedex, France \\ ${ }^{\mathrm{c}}$ Service de Radiologie A, Nouvel Hôpital Civil, Hôpitaux Universitaires de \\ Strasbourg, 1 place de l'Hôpital, BP 426, 67091 Strasbourg Cedex, France
}

\begin{abstract}
In this article, we propose an automatic algorithm for coronary artery segmentation from 3D X-ray data sequences of a cardiac cycle (3D-CT scan, 64 detectors, 10 phases). This method is based on recent mathematical morphology techniques (some of them being extended in this article). It is also guided by anatomical knowledge, using discrete geometric tools to fit on the artery shape independently from any perturbation of the data. The application of the method on a validation dataset (60 images: 20 patients in 3 phases) led to $90 \%$ correct (and automatically obtained) segmentations, the $10 \%$ remaining cases corresponding to images where the SNR was very low.
\end{abstract}

Key words: coronary arteries, segmentation, hit-or-miss transform, region-growing.

\section{Introduction}

A fatty substance called plaque can build up in the coronary arteries, blocking or slowing the flow of blood oxygenating the heart muscle. This coronary heart disease can lead to serious health problems, such as angina (pain or pressure in

\footnotetext{
* Corresponding author.

Email address: cronse@unistra.fr (C. Ronse).
} 
the chest) and heart attack [1]. This frequent and serious pathology justifies the development of computer-aided diagnosis software, improving the consistency of such diagnoses (in particular by speeding it up and making it more reliable in ambiguous situations).

Traditionally, 2D X-ray angiography (i.e., the classical and ancient projective method) used to be performed to visualize the blood vessels (in particular in the brain and heart), and especially to diagnose pathologies of these vessels such as blocking caused by plaque building up.

Thanks to recent breakthroughs in 3D image acquisition, vascular diagnosis is now generally made by considering 3D angiographic images, e.g. magnetic resonance angiography, or X-ray Computed Tomography Angiography (CTA) data, while 2D X-ray angiography remains mandatory for interventional procedures (angioplasty or before by-pass surgery, for instance). Nevertheless, 3D CTA remains difficult to interpret, as the $3 \mathrm{D}$ vascular tree is often still visualized by a projection in 2D. For diagnosis and treatment of coronary artery pathologies, a real 3D visualization is however highly suitable. 3D reconstruction (i.e., segmentation and 3D surfacic visualization) of the coronary arteries from CTA can lead to higher accuracy and reproducibility in the diagnoses and to better precision than 2D X-ray angiography in the quantification of the severity of the diseases.

Vessel segmentation algorithms are then the key component of such automated radiological diagnosis systems. Segmentation strategies vary depending on several parameters including imaging modality, application domain or automation requirements. Among the various image processing concepts that have been involved in the development of such methods, mathematical morphology has recently proved its efficiency, e.g. for hepatic $[2,3]$ and cerebral $[4,5]$ vascular networks.

In this article, which is the sequel of preliminary works described in [6-8], we propose to use mathematical morphology concepts for the design of an automatic method for segmentation of coronary arteries in large 3D CTA. In order to be robust to noise and contrast variability of these data, the method is based on a set of a priori anatomical knowledge. From an image processing point of view, two methodologies (enabling the use of such knowledge) are combined: an improved grey-level hit-or-miss transform, and region-growing. The segmentation method is decomposed into three steps: $(i)$ detection of the heart zone, (ii) detection of the aorta by application of a novel extension of the grey-level hit-or-miss transform, namely the blur grey-level hit-or-miss transform (B GL HMT), followed by a region-growing, and (iii) detection of coronary arteries using a B GL HMT-based region-growing initialized from seeds obtained in the second step. 
The remainder of this article is organized as follows. In Section 2, a state of the art of vessel segmentation summarizes previous works, especially related to coronary artery segmentation and mathematical morphology-based methods. Section 3 provides some background notions related to mathematical morphology and involved in the proposed segmentation method (namely the hit-or-miss and blur hit-or-miss transforms). A description of coronary artery anatomy (justifying the anatomical knowledge involved in the method) and of the considered CTA images are proposed in Section 4. The segmentation method is detailed in Section 5, while Section 6 describes experimental results. Concluding remarks and further works are discussed in Section 7.

\section{Previous works on vessel segmentation}

The segmentation of vascular structures from 3D data (especially MR and CT angiography) is an active field of research since the beginning of the 90's. These intensive efforts are motivated by the possible use of such segmentation results e.g. for pathology detection and quantification, or for surgical planning.

Some surveys of vessel segmentation methods before 2004 can be found in $[9,10]$. It has to be noted that most of the major image processing and analysis concepts have been involved in the development of 3D vessel segmentation methods (and have often been mixed in order to improve their respective advantages). Non-exhaustively, one can cite: region-growing [11,4], deformable models $[12,13]$, statistical analysis [14,15], minimal path-finding [16,17], vessel tracking [18,19], differential analysis [20,21], or mathematical morphology (see Subsection 2.2).

Hereafter, we especially focus on the methods devoted to coronary artery segmentation (Subsection 2.1) and mathematical morphology-based 3D vessel segmentation methods (Subsection 2.2). These two topics are indeed the focus of this article.

\subsection{Segmentation of coronary arteries}

The purpose of coronary artery segmentation is to enable a better detection, quantification and analysis of vascular pathologies (directly, but also by developing anatomical models of coronary arterial trees for labelling purpose $[22,23])$.

The first experiments in coronary segmentation have been performed in the 80 's on $2 \mathrm{D}$ X-ray data. These early methods were designed to obtain $2 \mathrm{D}$ results 
[24-26]. Further efforts have then been devoted to obtain 3D reconstructions of coronary arteries from single $2 \mathrm{D}+\mathrm{t}$ (rotating) $[27,28]$ or biplane angiograms [29-31].

The recent availability of 3D CTA images has finally led to the development of $3 \mathrm{D}$ coronary segmentation from such data. These methods elicit an increasing interest in the medical community (see e.g. [32]). Recent works related to this topic have implemented several methodological solutions: topological thinning [33], particle filtering [34], graph-based analysis [35], fuzzy connectedness [36], vessel tracking and active contours (in the context of micro-CTA analysis) [37], minimal cost path computation (on pre-filtered data) [38,39]. Except in [39] (where it was used in pre-filtering steps), mathematical morphology has - to the best of our knowledge - never been considered for 3D coronary segmentation from 3D CTA.

\subsection{Vessel segmentation and mathematical morphology}

A possible approach for vessel segmentation is provided by mathematical morphology, which analyses images or objects by their interaction with shapes. Morphological operators apply to images basic shapes called structuring elements; they were first defined for binary images, then extended to grey-level images. Dilation and erosion are the two main blocks for constructing morphological operators, such as openings, closings and hit-or-miss transforms. A good introduction to morphological operators can be found in [40]. A precise exposition of the extension of dilations, erosions and hit-or-miss transforms to grey-level images can be found in [41]. A detailed presentation of morphological image processing and analysis is given by [2].

For a long time, mathematical morphology has only been involved in the development of medical image analysis methods by considering low-level imageprocessing operators (erosions, dilations, openings, closings, etc.) [42-44].

The ability of high-level operators to be efficiently considered for such applications has however been pointed out in recent works (e.g. vertebrae labelling in 3D CT data [45], or left ventricular myocardium segmentation from $3 \mathrm{D}+\mathrm{t}$ MR data [46]). In particular, mathematical morphology operators that have been involved in vessel segmentation methods include: watershed transform $[47,48]$, grey-level hit-or-miss transform [5,49], or connected filtering based on component-trees [50,51]. The usefulness of such operators is justified by their intrinsic capacity to model morphological information, and then to enable anatomical knowledge-guided approaches.

In this context of guidance by anatomical knowledge, the grey-level hit-or-miss transform (possibly mixed with a region-growing process [3]) is a powerful tool 
for vessel segmentation, which enables in particular to take advantage of information related to probabilistic and/or geometric a priori knowledge about the searched vascular structures (see e.g. [52] for portal vein entry characterisation, or [53] for cerebral vascular atlases). The method that we will describe in this article is based on these concepts.

\section{Theoretical background: Hit-or-miss transform}

\subsection{Binary hit-or-miss transform}

The hit-or-miss transform (HMT) in binary images is a classic mathematical morphology operator [2], that uses two structuring elements $A$ and $B$. The operation is made by testing two conditions: $A$ has to belong to the object, while $B$ has to belong to the background. More formally, the HMT transform by the pair $(A, B)$ associates to a binary image $X$ the set $X \otimes(A, B)$ of positions $p$ where the translate $A_{p}$ of $A$ fits inside $X$ and at the same time the translate $B_{p}$ of $B$ fits inside the complement $X^{c}$ of $X$ :

$$
\begin{aligned}
X \otimes(A, B) & =\left\{p \in E \mid A_{p} \subseteq X \text { and } B_{p} \subseteq X^{c}\right\} \\
& =(X \ominus A) \cap\left(X^{c} \ominus B\right) \\
& =(X \ominus A) \backslash(X \oplus \check{B}) .
\end{aligned}
$$

Here $\check{B}=\{-b \mid b \in B\}$ and $\ominus$ designates the erosion operation [2]: $X \ominus A=$ $\left\{p \in E \mid A_{p} \subseteq X\right\}$. An example of binary HMT is illustrated in Fig. 1.

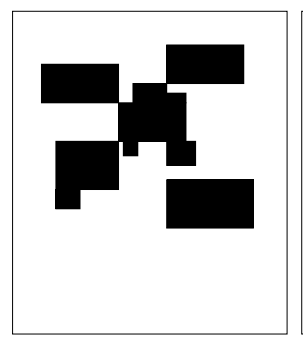

(a)

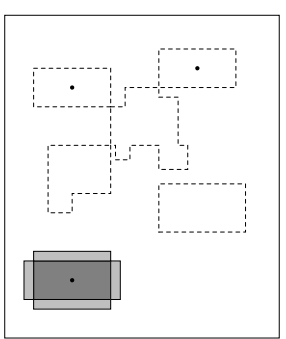

(b)

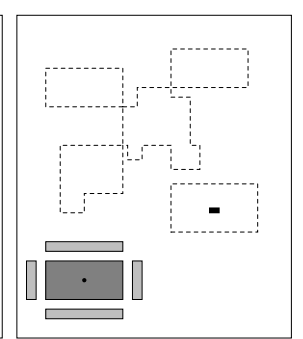

(c)

Fig. 1. Binary HMT. (a) Original image (object in black). (b) An example of HMT on (a): we illustrate bottom left the pair of foreground and background structuring elements (respectively in dark and light grey, the position of the origin being indicated by a black dot), the original image is indicated by its dotted border, and the result of the HMT consists of two black points. (c) Another example of HMT, where the two structuring elements have the same shapes, but not the same separation; here the HMT consists of a black rectangle. 


\subsection{Grey-level hit-or-miss transform}

The binary HMT operator is not easily extended to grey-level images. The various types of grey-level HMTs were unified in [41], and we have chosen as HMT the so-called supremal interval operator $\eta_{[V, W]}^{S}$. It uses two structuring functions $V$ and $W$ (respectively for foreground and background); given a grey-level image $I$, the value of $\eta_{[V, W]}^{S}(I)$ at each point $p$ is the greatest $t$ such that $V_{(p, t)} \leq I \leq W_{(p, t)}$, where $V_{(p, t)}$ and $W_{(p, t)}$ designate the translates of $V$ and $W$ by $p$ horizontally and by $t$ vertically; if no such $t$ exists, the value at $p$ will be the least grey-level $\perp$. Following [49], we choose $V$ having value $a$ on a support $A$ and $-\infty$ elsewhere, and $W$ having value $b$ on a dual support $B$ and $+\infty$ elsewhere (see Fig. 2(a)). Then we have:

$$
\begin{aligned}
& \eta_{[V, W]}^{S}(I)(p)= \\
& \begin{cases}(I \ominus A)(p)-a & \text { if }(I \ominus A)(p)-a \\
& \geq(I \oplus \check{B})(p)-b \\
\perp & \text { otherwise }\end{cases}
\end{aligned}
$$

Now we have:

$$
\begin{aligned}
& (I \ominus A)(p)=\min \left\{I(q) \mid q \in A_{p}\right\}=a_{\min } \\
& (I \oplus \check{B})(p)=\max \left\{I(q) \mid q \in B_{p}\right\}=b_{\max }
\end{aligned}
$$

In other words the grey-level HMT (GL HMT) compares the minimum intensity $a_{\min }$ in $A_{p}$ to the maximum intensity $b_{\max }$ in $B_{p}$; if $a_{\min }-b_{\max } \geq a-b$, then the point $p$ is selected and gets the grey-level $a_{m i n}-a$. This is illustrated in Fig. 2(b). For more technical details, the reader is referred to [41].

Two examples of GL HMT are illustrated in Figs. 3 and 4. The GL HMT is devoted to the detection of patterns which can be discriminated by their shape (that is, by their geometrical properties), the shape of their background, and the intensity difference between them. As we see in Figs. 3 and 4, the structuring elements can be used to detect in an image all locations where the grey-level on the object is higher than the one on the background by at least some height $h$ : here the height $h$ represents the minimum difference between the grey-levels of the foreground and background structuring elements at the given location. 


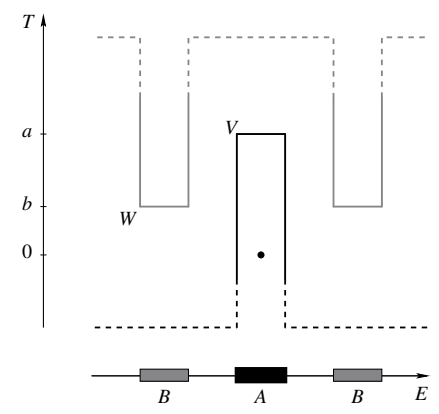

(a)

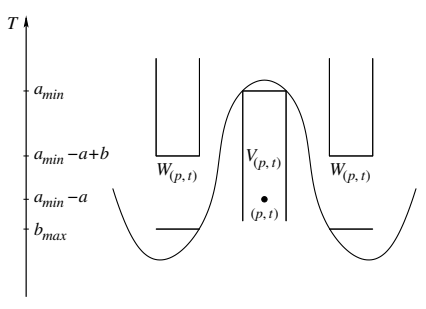

(b)

Fig. 2. (a) Given the two structuring elements $A$ and $B$ and the two grey-levels $a$ and $b$, the foreground structuring function $V$ has value $a$ on $A$ and $-\infty$ elsewhere, while the background structuring function $W$ has value $b$ on $B$ and $+\infty$ elsewhere. (b) Given $a_{m i n}$, the minimum grey-level of $I$ in $A_{p}$ and $b_{\text {max }}$, the maximum grey-level of $I$ in $B_{p}$, we have $a_{\min }-a+b \geq b_{\max }$, so the point $p$ is selected with grey-level $a_{\min }-a$.

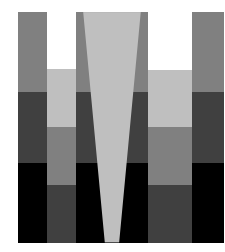

(a)

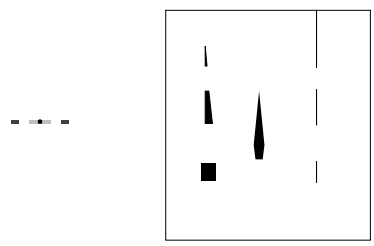

(c)

Fig. 3. Example of grey-level HMT application. (a) Initial grey-level image, with five different values, from 0 (black) to 4 (white). (b) The considered structuring elements: the foreground structuring element has value 3 (light grey) while the background structuring element has value 1 (dark grey); the position of the origin is indicated as a black dot (in the foreground structuring element). (c) Result of the GL HMT involving (a) and (b).

\subsection{Blur hit-or-miss transform}

Although the HMT is sensitive to the types of noise found in scanned images, including both boundary and random noise, a simple extension, the blur HMT, is relatively robust. The noise immunity of the blur HMT derives from its ability to deal with all forms of noise together, and to remove them by appropriate dilation or erosion. Thus the blur HMT (B HMT) can be used as 


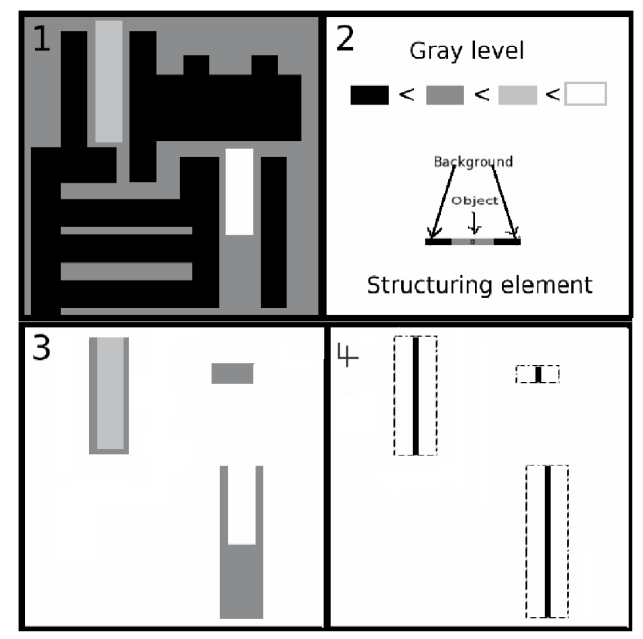

Fig. 4. Another example of grey-level HMT application : (1) Initial grey-level image, with four different values, from 0 (black) to 3 (white). (2) The foreground structuring element has value 2 (light grey) while the background structuring element has value 0 (black); the position of the origin is indicated as a black dot (in the foreground structuring element). (3) Positions where the foreground structuring element fits in the image. (4) Result of the GL HMT.

a fast heuristic to avoid more expensive integer-based matching techniques, and it is implemented efficiently with morphological image operators.

The B HMT for binary images was introduced in [54]. In the binary HMT, cf. (1), a point $p$ may not belong to $X \otimes(A, B)$ because of small holes or narrowings in $X$ or $X^{c}$, due to noise. They can be eliminated by dilating the foreground $X$ and background $X^{c}$ by two structuring element $G$ and $H$ that depend on image noise and pattern variability. Thus we compute

$$
\begin{aligned}
& X \otimes(A, B ; G, H) \\
& =\left\{p \in E \mid A_{p} \subseteq X \oplus G \text { and } B_{p} \subseteq X^{c} \oplus H\right\} \\
& =((X \oplus G) \ominus A) \cap\left(\left(X^{c} \oplus H\right) \ominus B\right) \\
& =((X \oplus G) \ominus A) \backslash((X \ominus \check{H}) \oplus \check{B}) .
\end{aligned}
$$

In the grey-level case, the dilation of the dark background is obtained by eroding the image. Here $G$ and $H$ are flat structuring elements. Thus we compute for an image $I$ the following blur GL HMT (B GL HMT):

$$
\begin{aligned}
& \eta_{[V, W ; G, H]}^{S}(I)(p)= \\
& \begin{cases}((I \oplus G) \ominus A)(p)-a & \text { if }((I \oplus G) \ominus A)(p)-a \\
& \geq((I \ominus \check{H}) \oplus \check{B})(p)-b \\
\perp & \text { otherwise } .\end{cases}
\end{aligned}
$$


Indeed, the dilation $I \oplus G$ eliminates dark noise in the bright foreground, while the erosion $I \ominus H$ eliminates bright noise in the dark background. Often one can take the two smoothing structuring elements equal, $G=H$, and symmetrical, $\check{G}=G$.

In this article, we will apply the B GL HMT to noisy 3D grey-level images obtained from heart scanners of several patients.

\subsection{Hit-or-miss opening}

The hit-or-miss transform detects all positions where the two structuring elements fit in the object and background respectively. In some situations, the goal is to show all object structuring elements fitting at these positions. This is obtained by following the HMT with a dilation by the object structuring element. In the binary case (1), the set $X$ is transformed into $(X \otimes(A, B)) \oplus A$, while in the grey-level case (2), the image $I$ becomes $\eta_{[V, W]}^{S}(I) \oplus V$. This operator is called the hit-or-miss opening (HMO) [41]. The difference between the HMO and the HMT is that in the HMO the searched structures are shown with their original width and grey-level, that were reduced in the HMT.

In this article, we will extract arteries by applying blur grey-level hit-or-miss openings (B GL HMO) $I \mapsto \eta_{[V, W ; G, H]}^{S}(I) \oplus V$, where $V$ is a structuring element $A$ at grey-level $a$ and $W$ a structuring element $B$ at grey-level $b$ (cf. Fig. 2), with variable sizes, orientations (for $B$ ), and contrasts $a-b$.

\section{Computed tomography angiography and cardio-vascular anatomy}

\subsection{Computed tomography angiography}

The images that we will analyse are CTA (3D X-ray) data visualizing the heart region, and especially the vascular structures. The quality of the images can suffer when the heart muscle is overlapped by the diaphragm or the ribs. The scan is performed with a contrast agent (iodine) using a technique called Bolus Tracking, or First Pass. The contrast material will be injected into a vein in the arm, using an automatic injector machine. Because the iodine is injected into a vein, CTA angiography could be considered as not invasive. These images have a submillimetric resolution $(0.6 \times 0.6 \times 0.6$ to $1.2 \mathrm{~mm})$, resulting in a large size $\left(512^{3}\right)$, and are generally anisotropic on the axial dimension. In addition to the heart, these images visualize a large part of the trunk, including the lungs, the spinal column, the liver and a part of the 
stomach (see Figs. 6(a) and 8). The data corresponding to each patient is composed of a series of 3D images, forming thus a 4D image of the cardiac cycle, divided into ten phases. The choice of a good phase where the coronary arteries are easily detected, depends on their size, which is generally larger in the 3 last phases, corresponding to the diastole.

\subsection{Cardio-vascular anatomy: coronary arteries}

Because the heart is composed primarily of cardiac muscle tissue that continuously contracts and relaxes, it must have a constant supply of oxygen and nutrients. The coronary arteries are the network of blood vessels that carry oxygen- and nutrient-rich blood to the cardiac muscle tissue.

The blood leaving the left ventricle exits through the aorta, the body's main artery. From the aorta generate the two coronary arteries, referred to as the left coronary artery (LCA) and the right coronary artery (RCA), they emerge from the beginning of the aorta, near the top of the heart (Fig. 5).

The initial segment of the left coronary artery is called the left main coronary. This blood vessel is approximately $4 \mathrm{~mm}$ wide and is less than $25 \mathrm{~mm}$ long. It branches into two slightly smaller arteries: the left anterior descending coronary artery (LAD) and the left circumflex coronary artery (LCX). The left anterior descending coronary artery is embedded in the surface of the front side of the heart. The left circumflex coronary artery circles around the left side of the heart and is embedded in the surface of the back of the heart.

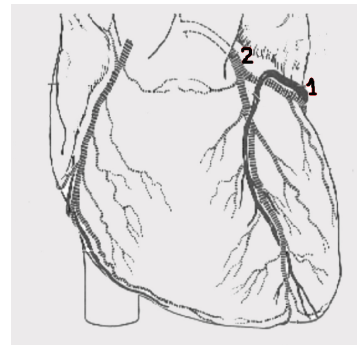

(a)

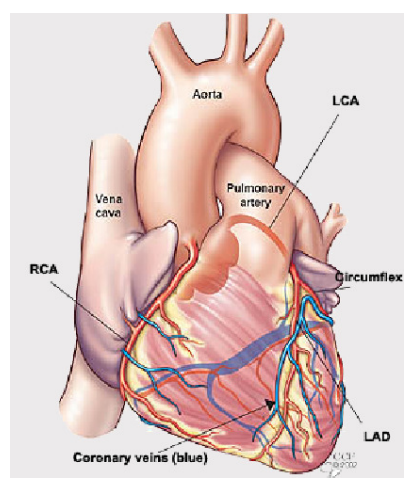

(b)

Fig. 5. (a) Heart anatomy: (1) coronary vein and (2) coronary artery. (b) Coronary artery anatomy. 


\section{Segmentation method}

\subsection{Input/output}

The method requires as input a $4 \mathrm{D}(3 \mathrm{D}+\mathrm{t})$ CTA image covering the cardiac cycle on ten phases. The coronary arteries have varying dimensions and do not introduce the same contrast on all stages of cardiac cycle. An optimum choice of the phases in which to perform segmentation proves to be necessary. Constriction and dilation of the coronary arteries, governed primarily by local regulatory mechanisms, regulate the amount of blood flow to the myocardium. During the diastole, the coronary arteries are the most dilated, so they present a better size and contrast in that stage. The diastole corresponds to the 7th, 8th and 9th phases of the 4D image. We are therefore going to choose these three phases to search the coronary arteries, and keep the best segmentation.

Selecting one of these phases of the heart, we obtain a 3D image $I$ similar to those presented previously. This image can be considered as a function $I: E \rightarrow \mathbb{Z}$ (with $E=[0,511]^{3}$ ), associating to each point $x \in E$ its grey-level $I(x)$. Since the method is fully automatic, no other parameter is required. The method provides as output a binary image $S \subset E$ corresponding to the segmented coronary arteries detected in $I$.

\subsection{Heart zone detection}

As described above, the image $I$ has huge dimensions, while the coronary arteries which have to be segmented from it are small structures. Consequently, their segmentation from $I$ without any pre-processing step will necessarily result in a prohibitive computational cost. In order to reduce this cost, two strategies can be considered: $(i)$ working on a sub-sampled image of $I$ whenever it is possible, and ( $i i)$ reducing the region of $I$ where the coronary arteries are supposed to be localized.

Until starting the actual segmentation of the coronary arteries (Subsection 5.3.3), all steps of the method (which do not require a fine resolution of the image) can be performed on a subresolution version $I_{s}$ of $I$. This image $I_{s}: E_{s} \rightarrow \mathbb{Z}$ is defined by $I_{s}(x)=\min \left\{I(y) \mid y \in 4 . x+[0,3]^{3}\right\}$, it is an approximation of $I$ where the high intensity small details have been removed.

The computational cost reduction induced by this sub-sampling can be optimized by also determining a region of interest of $I_{s}$ where the vascular structures will be located, namely the heart region. The idea is then to use a GL HMT (2) considering the lungs as background and the heart as object. The 
heart zone is detected with two ad hoc structuring elements: a sphere for $A$, and for $B$ one of two ellipsoids, where the vertical axis is longer than the horizontal ones, and smaller than the radius of $A$, and whose centers are horizontally symmetrical w.r.t. the center of $A$ (Fig. 6(b)). After studying a representative number of images, we have an average of difference of intensity in the order of the value 200. The GL HMO results in a subset $S_{s}^{h} \subset E_{s}=[0,127]^{3}$, which corresponds to the heart region (Fig. 6(c)).

As result, the search zone is reduced to one third (in each dimension) comparing to the original size, and then we can start the coronary artery segmentation. When we start the search for a thinner and smaller object, we need a high resolution, so it becomes necessary to return to the original size. We realize thus an over-sampling of the heart zone to go back to the original size $\left(512^{3}\right)$. An over-sampled version $S^{h}$ of $S_{s}^{h}$ is then obtained as $S^{h}=\bigcup_{x \in E}\left(4 . x+[0,3]^{3}\right)$.

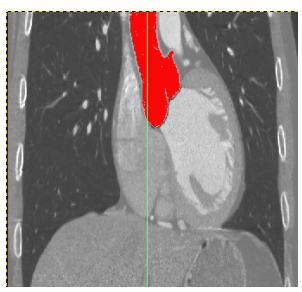

(a)

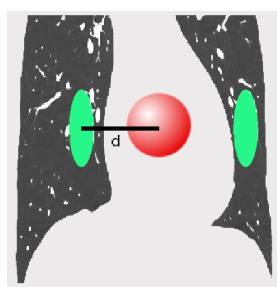

(b)

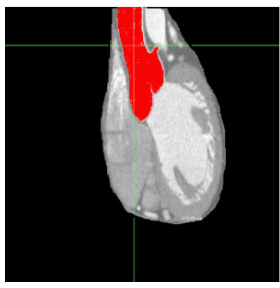

(c)

Fig. 6. Heart zone detection. (a) Original image. (b) Structuring elements used for the heart zone detection. (c) Heart zone.

\subsection{Coronary artery detection}

\subsubsection{Image pre-processing}

The processed images have a very low quality, a high noise and strong variance; thus a pre-processing is necessary to eliminate the effect of noise, especially of the "salt and pepper" type, that distorts the result of the HMT. This type of noise is a random process, due to scanner defects. A frequently observed defect is the occurrence of a dark noisy pixel ("pepper") inside a bright (foreground) zone, or of a bright noisy pixel ("salt") inside a dark (background) zone. This prevents the fitting of the structuring elements at required heights (cf. Fig. 2) leading thus to a non-detection by the GL HMT. Note however that the occurrence of a dark noisy pixel inside a dark zone, or of a bright noisy pixel inside a bright zone, does not hinder the working of the GL HMT.

The pre-processing consists in filtering these images, and it produces two new images: $I_{B}$ for the background freed from "salt" noise, and $I_{F}$ for the foreground freed from "pepper" noise (see Fig. 7). We use a filter with a window forming a $4^{3}$-cube; for $I_{B}$ we keep the lowest intensity, $I_{B}(x)=$ 
$\min \left\{I(y) \mid y \in x+[0,3]^{3}\right\}$, while for $I_{F}$ we keep the highest intensity, $I_{F}(x)=\max \left\{I(y) \mid y \in x+[0,3]^{3}\right\}$. Then we will dilate $I_{B}$ and erode $I_{F}$, as required by the blur GL HMT, see (4). Thus we apply the GL HMT, cf. (2), with two images instead of only one: at each location, the foreground structuring element has to fit in $I_{F}$ (Fig. $\left.7(\mathrm{~b})\right)$ and the background structuring element has to fit in $I_{B}$ (Fig. $7(\mathrm{c})$ ); if the difference of grey-levels $a_{\min }-b_{\max }$ exceeds the required minimum $a-b$, the point is selected.

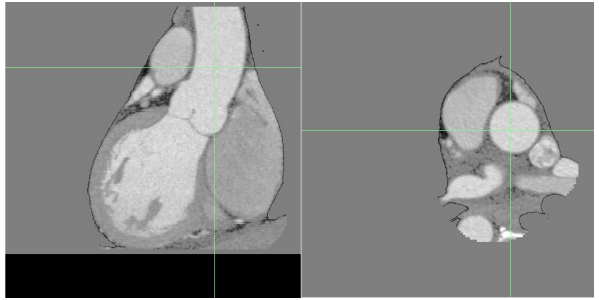

(a)

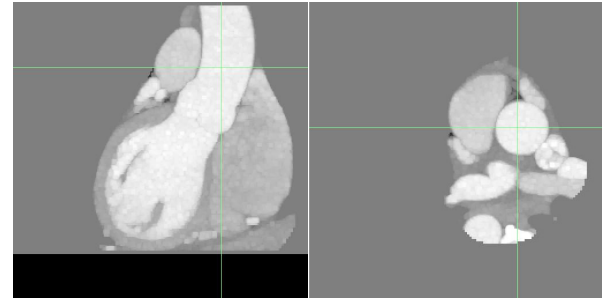

(b)

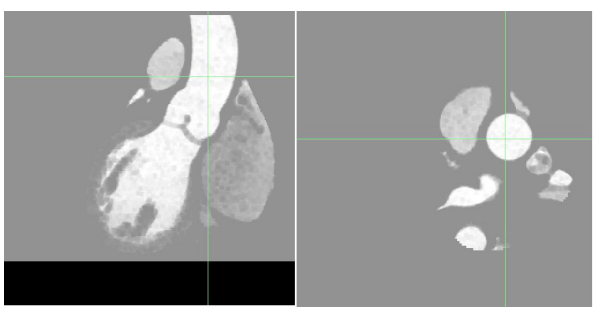

(c)

Fig. 7. Image pre-processing. (a) Original image. (b) High intensity dilated image. (c) Low intensity eroded image.

A naive way to segment coronary arteries could be a straightforward application of B GL HMO using structuring elements modelling the tubular structures and their background. This would detect not only the coronary arteries, but also other vessels of the heart, such as the coronary veins which are close to coronary arteries (Fig. 5). In order to avoid such problems and to efficiently perform segmentation, a solution, described below, consists in taking advantage of a priori anatomic knowledge related to the coronary arteries, in particular their size, their position (near the heart surface), and their initial point (on the aorta), and to design a segmentation strategy in adequation with this knowledge.

The main idea is to detect the coronary arteries using their tree structure, by applying region growing. Basically the method should be completely automatic, there has to be no user interaction; thus to realize the region growing, the seed-point detection has to be performed automatically. Once again we explore the anatomy of the heart, and as said above, the aorta can be considered as the seed region from which originate coronary arteries. So a first step will be to detect the beginning of the aorta from which generate these arteries. 


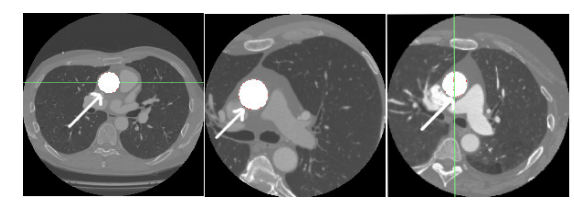

Fig. 8. Three examples of the aorta horizontal axial section.

\subsubsection{Aorta automatic detection}

About 2 to $3 \mathrm{~cm}$ above the aortic valve, an axial section of the aorta presents a circular shape (Fig. 8), with a radius varying from $1 \mathrm{~cm}$ to $2 \mathrm{~cm}$, this value depending on the patient and the conditions of the image acquisition. Starting from the previously obtained subset $S^{h}$ (corresponding to the heart region) we create a new subset $S^{a}$ representing the aorta. Our goal is to locate this circular shape using a B GL HMO with multi-sized structuring elements. A horizontal disk $A$ with a variable radius models the object. To model the background, we surround this disk with 8 points regularly sampled on a discrete circle $B$ (Fig. 9). Thus we will apply a B GL HMO with 3 pairs $(A, B)$ of structuring elements with varying sizes.

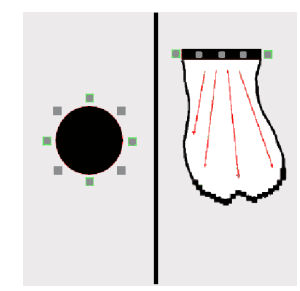

Fig. 9. Aorta segmentation. Left: Structuring elements used for the circular axial section detection. Right: region growing.

It is easy to note that in the original image, the dark space where the background structuring element should fit is relatively narrow, thus the pre-processing performed above (Fig. 7(c)) would enable to widen this region before we apply the B GL HMO using the structuring elements described above. Once this circular shape is detected, we calculate the average value of the intensities on this cut. The center of the detected circular shape will be used as seed for a first region-growing, the expansion criterion of which will involve two parameters: intensity thresholding and variance. This region-growing will evolve in the direction of the beginning of the aorta, and it will be stopped by the aorta wall on the sides and by the aortic valve below. The B GL HMO followed by the region-growing results in the subset $S^{a} \subset S^{h}$ which corresponds to the aorta segment (see e.g. Fig. 6(a,c), where the aorta segmentation result is shown in red). 


\subsubsection{Coronary artery seed-point detection}

The coronary arteries are the only tubular structures that arise from the beginning of the aorta. The idea is to search them in the wall of the aorta.

Following a strategy close to the one proposed in [52,3], a B GL HMT is applied, with a sphere as object and 8 points surrounding this sphere as background. These points belong to the plane normal to the artery axis. The choice of these points defines the axis direction of the searched coronary artery. Thus 13 structuring elements, corresponding to the 13 discrete principal directions, are used (Fig. 10).

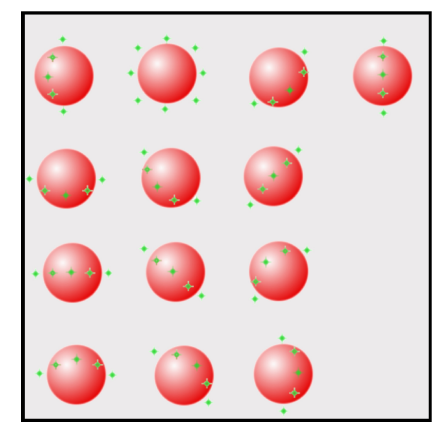

Fig. 10. Structuring element used for the coronary artery detection.

This B GL HMT is applied on the aorta wall, thus we obtain two seed-points $s_{l}=\left(x_{l}, y_{l}, z_{l}\right)$ and $s_{r}=\left(x_{r}, y_{r}, z_{r}\right) \in S^{a}$ for the left and right coronary arteries.

\subsubsection{Region-growing for coronary artery detection}

Starting from the two seed-points $s_{r}$ and $s_{l}$ previously detected on the aorta wall, a region-growing is performed, applying the B GL HMO on every neighbour as criterion. The involved structuring elements are the same as those described in the previous subsection for the seed-point detection; however for each of the 13 orientations, we take pairs of 4 different radii. The result is a subset $S \subset E$ which corresponds to the coronary arteries. An example of result (aorta and coronary arteries) is illustrated in Fig. 11(a).

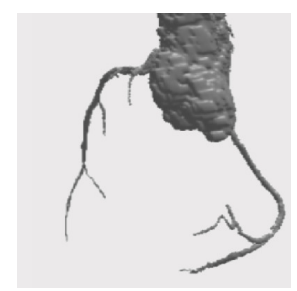

(a)

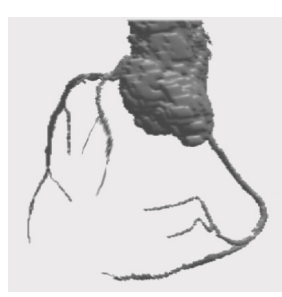

(b)

Fig. 11. Coronary artery segmentation. (a) Results with the B GL HMO. (b) Results with the Adaptive B GL HMO. 
Using the B GL HMO with a fixed grey-level difference between the foreground and the background does not allow the region growth to go far enough in the arteries. The more we advance in the coronary arteries, the darker they become, because of their decreasing size and the lower concentration of the injected contrast product. In the small bifurcations, their contrast with the cardiac muscle is less important than at the beginning of the artery.

An adaptive application of the B GL HMO during the region growing is necessary, in order to detect the greatest part of this artery. The adaptive aspect will consist in the reduction of the grey-level difference defined in the B GL HMT; during the region growth, each time that the B GL HMT fails, this difference is reduced by a small amount, while the structuring elements keep the same geometric form. We consider 3 values for that grey-level difference; thus the number of pairs of structuring elements tested at each position is at most 156 (13 orientations times 4 sizes times 3 contrasts). This region growth stops when, after 2 successive reductions of the difference, the B GL HMT still fails.

We have thus applied an Adaptive B GL HMO, that gives better results and makes the method more efficient (see Fig. 11(b)).

\subsection{Final algorithm}

The whole algorithm is summarized hereafter.

Input: Original image: $I: E \rightarrow \mathbb{Z}$ with $E=[0,511]^{3}$.

- Pre-processing:

- Under-sampling to get a $128^{3}$ image:

$I_{s}(x)=\min \left\{I(y) \mid y \in 4 . x+[0,3]^{3}\right\}$.

- Heart zone detection by GL HMO application: $S_{s}^{h} \subset E_{s}$.

- High intensity filter application and dilation to get: $I_{F}(x)=\max \{I(y) \mid y \in$ $\left.x+[0,3]^{3}\right\}$ dilated.

- Low intensity filter application and erosion to get: $I_{B}(x)=\min \{I(y) \mid y \in$ $\left.x+[0,3]^{3}\right\}$ eroded.

- Coronary artery segmentation:

- Aorta segmentation: $S_{s}^{a} \subset E$.

Circular transverse section localisation.

Computation of the average intensity on this section.

Region-growing starting by the circular shape center and using the calculated average intensity as criterion, in the direction of the beginning of the aorta.

- Over-sampling of the aorta to a $512^{3}$ image: $S^{a} \subset E$. 
- Search of the two coronary artery seed-points on the aorta wall: $s_{r}$ and $s_{l}$.

- Coronary artery detection with a region-growing using the Adaptive B GL HMO as criterion: $S \subset E$.

Output: Coronary artery (binary) image: $S \subset E$.

\section{$6 \quad$ Experiments and results}

The way to validate methods devoted to the extraction of coronary arteries depends on several criteria, including their actual purpose, the kind of data to be processed and the availability of a ground truth. In the literature, such validations were performed on databases containing from less than 6 images $[33,35,37,39]$ to approximately 35 images $[34,36]$.

It has to be noticed that, in general, validations are not performed by comparison to results obtained from other methods (except in few cases involving methods developed by the same authors, e.g. in [36] where comparisons however only deal with computation times). This can be explained by the difficulty to determine some comparison criteria between methods which present quite heterogeneous characteristics (automated methods [33,35,37] vs. interactive ones $[34,36,38,39]$, segmentation methods [34,36,37] vs. methods focusing on skeletonisation or linear structure extraction $[33,35,38,39]$, methods computing "classical" CT data [33-36,38,39] vs. methods devoted to new modalities [37], etc.).

Except in specific cases (e.g., in [39], where synthetic data were involved to assess the quality of centerline extractions, and in [37] where validations on synthetic data were required due to the huge amount of vessels visualised in the real images), the main way to validate the quality of the results still remains their visual inspection by an expert radiologist or cardiologist [34-36] or their comparison to manually obtained results [38]. Following this standard modus operandi, the validations described hereafter are based on a comparison with manually obtained results provided by an expert radiologist or cardiologist.

The method was tested on $60 \mathrm{CT}$ coronary angiographic images, for the 3 chosen phases of 20 patients. These patients had led to satisfactory diagnostic interpretations during the clinical examination in the scanner, where the segmentation of the coronary arteries was accomplished in an interactive mode by the cardiologist.

The first part of the method (the heart zone detection) was validated on 20 other patients. For the second part (aorta detection), some dimension had to be set, namely the radius of the horizontal disk modelling the aorta section. 
Several radii were tried on a set of images, and for each image the best radius was selected. We obtained thus a range of radii for the whole base. This enables to adjust the radius to each image of the base.

According to the result of the segmentation algorithm, for each patient, only one of the 3 phases is taken into account: the one where the coronary arteries are best visualized. We can separate our results into three groups. A first group composed of $50 \%$ of the tested images gives visually satisfactory results. A second group composed of $40 \%$ of the images provides less satisfactory results, the region growth in the last step (for segmenting the coronary arteries) has been diverted to give false positives, in most cases consisting of the wall of the heart. Thus a post-processing of this group of images becomes necessary, it consists in adding a constraint on the GL HMT, namely the percentage of accepted voxels in the object structuring element: we require $90 \%$ of these voxels to satisfy the difference of grey-level between them and the maximum grey-level of the background (Fig. 12); note that this added constraint has no effects on the first group of images that already had satisfactory results. The third group consists of the remaining 10\% images, they are noisier and their variance is higher than that of the others; thus some of the coronary arteries in these images are completely blurred and present no contrast with the background, which makes the application of the automatic segmentation just impossible.

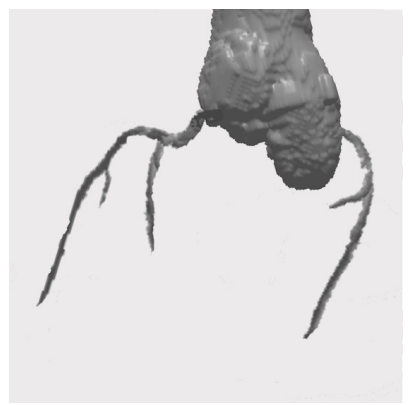

(a)

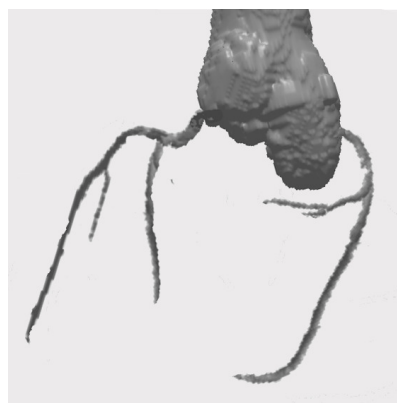

(b)

Fig. 12. Example of segmented image: (a) without post-processing; (b) with post-processing.

Finally we got legible results for $90 \%$ of our image base. In this group of images we observed that one third of them did not contain the circumflex (LCX) branch. The detection of the first bifurcation was not performed because the maximum size of the structuring element was smaller than that of the artery bifurcation. An increase of that size was enough to solve this problem.

The final results were validated by an expert cardiologist. His validation confirmed that $90 \%$ of the cases were very well segmented (from the heart zone to the coronary arteries). A typical example of segmentation is shown in Fig. 13, where the various branches are labelled with their customary abbreviations. 


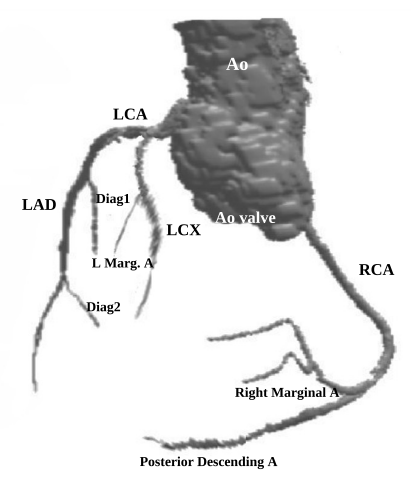

Fig. 13. Segmented coronary tree.

This expert considered that the non-validated $10 \%$ concern two patients presenting important calcification, in the presence of stent, with a low image quality compared to the other $90 \%$. The diagnostic exploitation of these two patients was also very difficult with a manual segmentation in the scanner. Our automatic method detected for the 18 other cases the left main coronary artery (LCA) and the left anterior descending artery (LAD). In only one case the right coronary artery (RCA) was not detected, because it was abnormally small. The first diagonal branch (Diag1) was not detected in 3 cases, among which 2 cases were also ignored by the manual segmentation of the cardiologist.

In a few cases of our base, the automatic detection is more efficient than the manual segmentation: the first diagonal branch (Diag1) and right marginal branch are located by our method, but not detected by the cardiologist through manual segmentation.

Note that the presence of stents did not perturb our segmentation, except in the case of important calcification. Our segmentation algorithm is also robust under anomalies such as variation in the relative sizes of the various coronary arteries, or the origin of the RCA being near that of the LCA.

\section{Conclusion and future work}

We have proposed a method for 3D segmentation of coronaries. This method is automatic in the sense that it does not require a manual placement of markers or user interaction (however in the experiments, some dimensions corresponding to vessel sizes had to be adjusted, but for adult patients they usually depend only on the image resolution). It has been validated by an expert on a database of 20 patients, giving correct results for $90 \%$ of them; the remaining $10 \%$ are low-quality images, presenting difficulties for the expert too. 
Further details on the scientific context, the working of our segmentation algorithms and the experimental results can be found in the first author's Ph.D. thesis [55].

From the mathematical point of view, this work illustrates the potential of the grey-level hit-or-miss transform [41,49] and its variants [54] for the segmentation of angiographic images. We feel that the merits of this type of operator have not yet been fully recognized in this context.

A study will be undertaken on the behaviour of the coronary arteries in the 4th dimension: time. In order to realize this study, we have to adapt this method to the 4th dimension, independently from the data changes from a case to another, and even from a phase to the next one in the same case.

\section{References}

[1] C. O'Callaghan et al., Stress and the Heart: Psychosocial Pathways to Coronary Heart Disease, Wiley, 2002.

[2] P. Soille, Morphological Image Analysis, 2nd Edition, Springer-Verlag, Heidelberg, 2003.

[3] B. Naegel, C. Ronse, L. Soler, Using grey-scale hit-or-miss transform for segmenting the portal network of the liver, in: ISMM 2005, Vol. 30 of Computational Imaging and Vision, Springer SBM, 2005, pp. 429-440.

[4] N. Passat, C. Ronse, J. Baruthio, J.-P. Armspach, C. Maillot, C. Jahn, Regiongrowing segmentation of brain vessels: An atlas-based automatic approach, Journal of Magnetic Resonance Imaging 21 (6) (2005) 715-725.

[5] N. Passat, C. Ronse, J. Baruthio, J.-P. Armspach, C. Maillot, Magnetic resonance angiography: From anatomical knowledge modeling to vessel segmentation, Medical Image Analysis 10 (2) (2006) 259-274.

[6] B. Bouraoui, C. Ronse, J. Baruthio, N. Passat, P. Germain, Gray-level hit-ormiss transform based region-growing for automatic segmentation of 3D coronary arteries, in: ISMM 2007, Vol. 2, INPE, 2007, pp. 23-24.

[7] B. Bouraoui, C. Ronse, J. Baruthio, N. Passat, P. Germain, Fully automatic 3D segmentation of coronary arteries based on mathematical morphology, in: ISBI 2008, 2008, pp. 1059-1062.

[8] B. Bouraoui, C. Ronse, J. Baruthio, N. Passat, P. Germain, Blur greylevel hit-or-miss transform for fully automatic 3D segmentation of coronary arteries, in: M. Wilkinson, J. Roerdink (Eds.), 9th International Symposium on Mathematical Morphology (ISMM), Abstract Book, University of Groningen, 2009, pp. 37-40. 
[9] J. Suri, K. Liu, L. Reden, S. Laxminarayan, A review on MR vascular image processing: Skeleton versus nonskeleton approaches: Part II, IEEE Transactions on Information Technology in Biomedicine 6 (4) (2002) 338-350.

[10] C. Kirbas, F. Quek, A review of vessel extraction techniques and algorithms, ACM Computing Surveys 36 (2) (2004) 81-121.

[11] X. Tizon, Ö. Smedby, Segmentation with gray-scale connectedness can separate arteries and veins in MRA, Journal of Magnetic Resonance Imaging 15 (4) (2002) 438-445.

[12] L. Lorigo, O. Faugeras, W. Grimson, R. Keriven, R. Kikinis, A. Nabavi, C.F. Westin, CURVES: Curve evolution for vessel segmentation, Medical Image Analysis 5 (3) (2001) 195-206.

[13] M. Descoteaux, D. Collins, K. Siddiqi, A geometric flow for segmenting vasculature in proton-density weighted MRI, Medical Image Analysis 12 (4) (2008) 497-513.

[14] A. Chung, J. Noble, P. Summers, Vascular segmentation of phase contrast magnetic resonance angiograms based on statistical mixture modeling and local phase coherence, IEEE Transactions on Medical Imaging 23 (12) (2004) 14901507.

[15] M. Sabry Hassouna, A. Farag, S. Hushek, T. Moriarty, Cerebrovascular segmentation from TOF using stochastic models, Medical Image Analysis 10 (1) (2006) 2-18.

[16] H. Li, A. Yezzi, Vessels as 4-D curves: Global minimal 4-D paths to extract 3-D tubular surfaces and centerlines, IEEE Transactions on Medical Imaging 26 (9) (2007) 1213-1223.

[17] W. Wong, A. Chung, Probabilistic vessel axis tracing and its application to vessel segmentation with stream surfaces and minimum cost paths, Medical Image Analysis 11 (6) (2007) 567-587.

[18] N. Flasque, M. Desvignes, J.-M. Constans, M. Revenu, Acquisition, segmentation and tracking of the cerebral vascular tree on 3D magnetic resonance angiography images, Medical Image Analysis 5 (3) (2001) 173-183.

[19] R. Manniesing, M. Viergever, W. Niessen, Vessel axis tracking using topology constrained surface evolution, IEEE Transactions on Medical Imaging 26 (3) (2007) 309-316.

[20] Y. Sato, S. Nakajima, N. Shiraga, H. Atsumi, S. Yoshida, T. Koller, G. Gerig, R. Kikinis, Three-dimensional multi-scale line filter for segmentation and visualization of curvilinear structures in medical images, Medical Image Analysis 2 (2) (1998) 143-168.

[21] A. Frangi, W. Niessen, R. Hoogeveen, T. van Walsum, M. Viergever, Modelbased quantitation of 3-D magnetic resonance angiographic images, IEEE Transactions on Medical Imaging 18 (10) (1999) 946-956. 
[22] N. Ezquerra, S. Capell, L. Klein, P. Duijves, Model-guided labeling of coronary structure, IEEE Transactions on Medical Imaging 17 (3) (1998) 429-441.

[23] C. Chalopin, G. Finet, I. Magnin, Modeling the 3D coronary tree for labeling purposes, Medical Image Analysis 5 (4) (2001) 301-315.

[24] P. Eichel, E. Delp, K. Koral, A. Buda, A method for a fully automatic definition of coronary arterial edges from cineangiograms, IEEE Transactions on Medical Imaging 7 (4) (1988) 313-320.

[25] K. Kitamura, J. Tobis, J. Sklansky, Estimating the 3D skeletons and transverse areas of coronary arteries from biplane angiograms, IEEE Transactions on Medical Imaging 7 (3) (1988) 173-187.

[26] A. Klein, F. Lee, A. Amini, Quantitative coronary angiography with deformable spline models, IEEE Transactions on Medical Imaging 16 (5) (1997) 468-482.

[27] T. Nguyen, J. Sklansky, Reconstructing the 3-D medial axes of coronary arteries in single-view cineangiograms, IEEE Transactions on Medical Imaging 13 (1) (1994) 61-73.

[28] C. Blondel, G. Malandain, R. Vaillant, N. Ayache, Reconstruction of coronary arteries from a single rotational X-ray projection sequence, IEEE Transactions on Medical Imaging 25 (5) (2006) 653-663.

[29] A. Wahle, E. Wellnhofer, I. Mugaragu, H. Saner, H. Oswald, E. Fleck, Assessment of diffuse coronary artery disease by quantitative analysis of coronary morphology based upon 3-D reconstruction from biplane angiograms, IEEE Transactions on Medical Imaging 14 (2) (1995) 230-241.

[30] S. Chen, J. Carroll, 3-D reconstruction of coronary arterial tree to optimize angiographic visualization, IEEE Transactions on Medical Imaging 19 (4) (2000) $318-336$.

[31] L. Sarry, J.-Y. Boire, Three-dimensional tracking of coronary arteries from biplane angiographic sequences using parametrically deformable models, IEEE Transactions on Medical Imaging 20 (12) (2001) 1341-1351.

[32] C. Metz, M. Schaap, T. van Walsum, A. van der Giessen, A. Weustink, N. Mollet, G. Krestin, W. Niessen, Editorial: 3D segmentation in the clinic: A grand challenge II - Coronary artery tracking, in: IJ - 2008 MICCAI Workshop - Grand Challenge Coronary Artery Tracking, Proceedings, 2008. URL http://hdl.handle.net/10380/1399

[33] Z. Chen, S. Molloi, Automatic 3D vascular tree construction in CT angiography, Computerized Medical Imaging and Graphics 27 (6) (2003) 469-479.

[34] C. Florin, N. Paragios, J. Williams, Particle filters, a quasi-Monte Carlo solution for segmentation of coronaries, in: MICCAI 2005, Vol. 3749 of Lecture Notes in Computer Science, Springer, 2005, pp. 246-253.

[35] A. Szymczak, A. Stillman, A. Tannenbaum, K. Mischaikow, Coronary vessel trees from 3D imagery: A topological approach, Medical Image Analysis 10 (4) (2006) 548-559. 
[36] C. Wang, Ö. Smedby, Coronary artery segmentation and skeletonization based on competing fuzzy connectedness tree, in: MICCAI 2007, Vol. 4791 of Lecture Notes in Computer Science, Springer, 2007, pp. 311-318.

[37] J. Lee, P. Beighley, E. Ritmana, N. Smith, Automatic segmentation of 3D microCT coronary vascular images, Medical Image Analysis 11 (6) (2007) 630-647.

[38] S. Olabarriaga, M. Breeuwer, W. Niessen, Minimum cost path algorithm for coronary artery central axis tracking in CT images, in: MICCAI 2003, Vol. 2879 of Lecture Notes in Computer Science, Springer, 2003, pp. 687-694.

[39] D. Mueller, A. Maeder, Robust semi-automated path extraction for visualising stenosis of the coronary arteries, Computerized Medical Imaging and Graphics 32 (6) (2008) 463-475.

[40] R. Jain, R. Kasturi, B. Schunck, Machine Vision, McGraw-Hill, Inc., New York, NY, USA, 1995.

[41] B. Naegel, N. Passat, C. Ronse, Grey-level hit-or-miss transforms - Part I: Unified theory, Pattern Recognition 40 (2) (2007) 635-647.

[42] B. Thackray, A. Nelson, Semi-automatic segmentation of vascular network images using a rotating structuring element (ROSE) with mathematical morphology and dual feature thresholding, IEEE Transactions on Medical Imaging 12 (3) (1993) 385-392.

[43] G. Gerig, T. Koller, G. Székely, C. Brechbühler, O. Kübler, Symbolic description of 3-D structures applied to cerebral vessel tree obtained from MR angiography volume data, in: IPMI 1993, Vol. 687 of Lecture Notes in Computer Science, Springer, 1993, pp. 94-111.

[44] H. Cline, D. Thedens, C. Meyer, D. Nishimura, T. Foo, S. Ludke, Combined connectivity and a gray-level morphological filter in magnetic resonance coronary angiography, Magnetic Resonance in Medicine 43 (6) (2000) 892-895.

[45] B. Naegel, Using mathematical morphology for the anatomical labeling of vertebrae from 3D CT-scan images, Computerized Medical Imaging and Graphics 31 (3) (2007) 141-156.

[46] J. Cousty, L. Najman, M. Couprie, Segmentation cardiaque spatio-temporelle, in: L. Najman, H. Talbot (Eds.), Morphologie Mathématique : approches nondéterministes, mise en œuvre et applications, Hermès, 2010, Ch. 10.

[47] R. Rodríguez, T. Alarcón, O. Pacheco, A new strategy to obtain robust markers for blood vessels segmentation by using the watersheds method, Computers in Biology and Medicine 35 (8) (2005) 665-686.

[48] N. Passat, C. Ronse, J. Baruthio, J.-P. Armspach, J. Foucher, Watershed and multimodal data for vessel segmentation: Application to the superior sagittal sinus, Image and Vision Computing 25 (4) (2007) 512-521.

[49] B. Naegel, N. Passat, C. Ronse, Grey-level hit-or-miss transforms - Part II: Application to angiographic image processing, Pattern Recognition 40 (2) (2007) 648-658. 
[50] M. Wilkinson, M. Westenberg, Shape preserving filament enhancement filtering, in: MICCAI 2001, Vol. 2208 of Lecture Notes in Computer Science, Springer, 2001, pp. $770-777$.

[51] B. Caldairou, B. Naegel, N. Passat, Segmentation of complex images based on component-trees: Methodological tools, in: ISMM 2009, Vol. 5720 of Lecture Notes in Computer Science, Springer, 2009, pp. 171-180.

[52] B. Naegel, C. Ronse, L. Soler, Segmentation automatique de l'entrée de la veine porte dans le foie par application de la transformée en tout-ou-rien en niveaux de gris, in: ORASIS 2003, 2003, pp. 33-37.

[53] N. Passat, C. Ronse, J. Baruthio, J.-P. Armspach, C. Maillot, Cerebral vascular atlas generation for anatomical knowledge modeling and segmentation purpose, in: CVPR 2005, Vol. 2, 2005, pp. 331-337.

[54] D. Bloomberg, L. Vincent, Pattern matching using the blur hit-miss transform, Journal of Electronic Imaging 9 (2) (2000) 140-150.

[55] B. Bouraoui, Segmentation automatique de l'arbre coronarien à partir d'images angiographiques $3 \mathrm{D}+\mathrm{t}$ de scanner, Ph.D. thesis, Université de Strasbourg (2009). 